\title{
Linx
}

Revue des linguistes de l'université Paris X Nanterre

$41 \mid 1999$

L'hypothétique

\section{A propos de repérages fictifs : variété des formes et construction du sens}

Anne Trévise

\section{(2) OpenEdition}

Journals

Édition électronique

URL : http://journals.openedition.org/linx/1166

DOI : 10.4000/linx.1166

ISSN : 2118-9692

Éditeur

Presses universitaires de Paris Nanterre

Édition imprimée

Date de publication : 1 décembre 1999

Pagination : 39-59

ISBN : 0246-8743

ISSN : 0246-8743

\section{Référence électronique}

Anne Trévise, «A propos de repérages fictifs : variété des formes et construction du sens », Linx [En ligne], 41 | 1999, mis en ligne le 27 août 2012, consulté le 19 avril 2019. URL : http://

journals.openedition.org/linx/1166; DOI : 10.4000/linx.1166

Ce document a été généré automatiquement le 19 avril 2019

Département de Sciences du langage, Université Paris Ouest 


\title{
A propos de repérages fictifs : variété des formes et construction du sens
}

\author{
Anne Trévise
}

1 Dans le domaine des constructions de repérages fictifs à l'œuvre dans l'activité langagière (très généralement associées à l'idée d'hypothétique, irréel ou potentiel), et des différents types de relations d'inférence entre une protase et une apodose (du type : si $\mathrm{p}$ alors $\mathrm{q}$ ), on est d'emblée confronté à tout un ensemble de problèmes complexes dont certains seulement seront effleurés ici ${ }^{1}$ dans le cadre d'une théorie des opérations énonciatives.

\section{Définition d'un sous-domaine : le potentiel et l'irréel}

2 L'hypothèse, comme l'étymologie le souligne, est une proposition posée en dessous, qui tient lieu de fondement, de repère, de base, à un raisonnement. Ce raisonnement se fait par la composition de deux relations prédicatives, $\mathrm{p}$ et $\mathrm{q}$, qui en hypotaxe apparaissent sous la forme d'une « subordonnée » et d'une " principale ».

3 Le domaine décrit ici recouvre les notions traditionnellement appelées «potentiel » et « irréel », avec pour exemples prototypiques, non ambigus grâce aux déictiques demain et hier:

S'il faisait beau demain, j'irais me promener / S'il fait beau demain, j'irai / je vais me promener (potentiel).

S'il avait fait beau hier, j'aurais été me promener / S'il faisait beau maintenant, j'irais me promener (irréel).

Ces deux exemples hypotaxiques, à protase en si antéposée, ne présentent aucune ambiguïté. Ils réfèrent à des situations particulières (par opposition à S'il faisait beau, j'allais me promener ou S'il fait beau, je vais me promener) même si la relation d'entraînement entre $\mathrm{p}$ et $\mathrm{q}$ repose sur une pertinence généralement admise dans nos sociétés: les notions complexes aisément identifiables <faire beau> et <aller se promener> sont banalement associées dans une relation orientée d'inférence de l'une à l'autre. 


\subsection{Repère fictif décroché et modalité}

5 A partir de cette protase $p$ qu'il choisit d'ériger en repère fictif, choisissant donc de basculer dans le domaine modal du fictif pour toutes sortes de raisons, l'énonciateur construit une relation d'inférence (pouvant être fondée sur différentes relations, plus ou moins admises dans les connaissances partagées, de cause, de conditionnement, de conséquence, etc., ou pouvant plus relever de son arbitraire, ou relater un autre arbitraire / libre arbitre). Cette relation d'inférence entraîne la prédication de la notion complexe qu'est l'apodose q <moi, aller promener, ne pas aller promener>. L'inférence, comme le souligne Culioli à propos du marqueur donc en français (1990: 175), est un repérage mixte mêlant concomitance (identification) et consécution (différenciation) dans la dynamique orientée d'une relation. La deuxième lexis, l'apodose dans le cas des protases antéposées, est ainsi repérée par rapport à la première qui est en quelque sorte vue comme causale.

6 On voit que dans les deux cas du potentiel et de l'irréel, l'énonciateur pose du fictif, en s'appuyant sur un préconstruit repère, qui est donc premier, et que représente, pour lui et pour le coénonciateur ${ }^{2}$, le domaine de l'effectif. La construction du sens et sa reconstruction par l'allocutaire vont devoir inclure le décrochage entre les deux plans, entre les deux ordres de modalité. De même que toute négation présuppose une valeur positive première à nier, tout décrochage fictif construit par une protase est d'abord repéré par rapport à l'effectif avant de devenir repère de la relation d'inférence qui va aboutir à la prédication de l'apodose.

\subsection{Opérations liées à l'irréel}

7 Pour l'irréel, depuis le repère décroché, fictif, l'énonciateur envisage la notion complexe $\mathrm{p}$, en sachant que c'est non $\mathrm{p}$ (non validation) qui est, ou a été, le cas. Il se remet, pour ainsi dire, grâce au repère fictif décroché, à un stade antérieur, où les bifurcations vers $p$ et vers non $\mathrm{p}$ étaient encore dans le domaine du possible, mais en sachant qu'une seule des deux branches a pu être le cas dans l'extralinguistique, révolu ou actuel (cf. Merle, 1998, 1999 et ici même). C'est là un autre type de préconstruit : ce qui a été le cas peut être reconstruit par l'allocutaire (une protase en Si p par exemple implique, avec l'irréel, que c'est non p qui a été ou est le cas. Il s'agit d'une opération complexe qui tient de la modalité de la négation, autre opération seconde). L'énonciateur projette alors la relation d'entraînement d'après sa connaissance du monde ou de la situation, ou en prenant le parti d'un certain arbitraire, et prédique la proposition q repérée qui représente donc elle aussi un état de choses qui n'a pas été, ou n'est pas, le cas, sauf dans des exemples du type :

S'il avait fait beau, je serais aussi / tout de même restée à la maison. où aussi et tout de même marquent que l'altération fictive n'a pas entraîné de conséquence pour q par rapport à l'effectif. P est inefficace quant à q.

\subsection{Opérations liées au potentiel}

8 Le schéma du repère décroché par rapport aux deux branches de la bifurcation notionnelle, menant à $\mathrm{p}$ et non $\mathrm{p}$ respectivement, est le même que pour l'irréel, mais les opérations effectuées ne sont pas identiques. A partir d'un repère subjectif décroché par 
rapport à l'effectif, plan repère premier, la protase $p$ est aussi construite à son tour comme un repère (fictif), mais les repérages ne sont pas faits par rapport à un effectif révolu ou actuel. Aucune des deux branches ne représente plus ce qui est ou a été le cas, comme pour l'irréel, et on est en présence de la bifurcation qui caractérise la référence à l'à-venir, le " potentiel », même s'il est parfois perçu comme quasiment impossible, avec donc une branche très peu envisageable, mais on ne sait jamais...

La prédication porte sur l'à-venir, de toute façon non certain, et peut souvent relever d'un plus grand arbitraire énonciatif, thématique ou situationnel que dans le cas de l'irréel. La relation d'inférence est soit fondée sur des connaissances physico-culturelles (les liens entre beau temps et promenade par exemple), soit elle relève de ce qui peut être perçu comme de l'arbitraire de l'énonciateur ou d'un tiers, dans des situations particulières (Ah tiens, si tu y penses, tu pourras m'acheter une baguette ?, etc.).

10 Avec le potentiel, il n'y a pas le même travail relevant de la négation qu'avec l'irréel. Les deux branches sont ouvertes sur l'à-venir, domaine du non certain, et envisagent donc les deux possibilités tout en n'en énonçant qu'une (sauf dans des cas particuliers comme Qu'il fasse beau ou pas demain, j'irai me promener, où l'inefficacité de p est soulignée).

11 Ce qui distingue par exemple une «circonstancielle» en quand d'une "conditionnelle» en si (même si les deux sont quasiment interchangeables dans les cas de générique ${ }^{3}$ - en allemand wenn a précisément les deux sens), c'est qu'avec cette dernière on envisage toujours l'altérité <faire beau, ne pas faire beau>, p et non p (cf. Merle 1998 et 1999, Culioli, 1999: 160-163). En français par exemple, imparfait et conditionnel sont des marqueurs de cette modalité de rupture par rapport aux coordonnées énonciatives. En anglais, cela pourra être le prétérit, et un auxiliaire modal de conséquence prévisible ( will / would) dans l'apodose.

Déjà insérée dans le domaine modal du non certain à-venir et du fictif, la branche choisie pour la prédication de $\mathrm{p}$ peut se voir attribuer une représentation en termes de probabilité plus ou moins grande (de par les marqueurs de temps / aspects choisis notamment), et l'autre branche peut s'effacer plus ou moins (grande probabilité de p), ou rester très présente comme alternative possible. Mais la spécificité du schéma, comme pour certains auxiliaires de modalité anglais - on pense à may bien sûr ${ }^{4}$-, est que l'énonciateur projette un état de choses sans jamais exclure son complémentaire, autre que $\mathrm{p}$ ou non $\mathrm{p}$.

13 A partir de cette projection, il construit une relation d'inférence qui elle-même est une projection : il projette donc $\mathrm{p}$ (sans écarter non $\mathrm{p}$ ), et à partir de ce repère, il effectue une projection hypothétique qui s'appuie sur une hypothèse ayant acquis le statut de repère après avoir été elle-même repérée. L'inférence doit ou peut aboutir à q, avec là aussi toutes les nuances possibles apportées par les différents marqueurs quant au probable, possible, ou seulement potentiel, mais aussi quant à la subjectivité et à l'intersubjectivité. On énonce q mais on n'écarte jamais totalement non q, ou autre que q, même si l'on n'en dit rien, puisque non p n'est pas écarté.

\subsection{Un schéma métalinguistique unique pour diverses opérations}

L'irréel et le potentiel sont des domaines de compositions de lexis à partir d'un repère fictif, et s'y attache tout un éventail de valuations subjectives et intersubjectives : regret, reproche, pour l'irréel par exemple, espoir, rêverie, souhait, conseil, menace ou promesse 
pour le potentiel. On construit le fictif et l'inférence qui s'y appuie pour servir des argumentations ou des stratégies intersubjectives très diverses. On voit la parenté avec les modalités épistémiques, déontiques et appréciatives ainsi que, dans certains emplois des auxiliaires modaux de l'anglais, l'intrication des valuations et des modulations (cf. Charreyre, 1984, Gresset, 1999).

C'est donc un champ sémantique complexe et ramifié qui s'exprime grâce à tout un emboîtement d'opérations prédicatives et énonciatives complexes, hors de l'assertion, et avec tous les paramètres inclus par les marqueurs employés (subordonnants éventuels, temps, aspect, jeu des négations, notions impliquées, ordre des prédications, et relations entre les notions complexes impliquées). Ces marqueurs interagissent dès lors qu'ils sont mis en relation les uns avec les autres pour construire le sens et le faire reconstruire.

Quels que soient les marqueurs employés, il y a en résumé une très nette différence entre ce qui peut encore être affecté par un changement, même si l'énonciateur n'y croit pas (le potentiel), souvent valué en positif ou en négatif (souhait, crainte), et ce qui ne peut être que du domaine du constat, valué ou non par du regret ou du reproche, par rapport à ce qui a été le cas (l'irréel), et qui constitue le préconstruit, préexistant aux relations d'entraînement et les fondant.

Le concept d'altérité est ici primordial dans la construction des représentations par les énonciateurs. Dans le domaine du fictif, on se représente un état des choses, en le posant le plus souvent explicitement comme fictif, par rapport à une "réalité». C'est une opération langagière complexe, que l'on peut donc schématiquement représenter, comme Culioli le fait, par un repère subjectif décroché à partir duquel on envisage les deux branches d'une bifurcation allant respectivement vers $p$ ou non $p$. Le chemin abstrait parcouru sur ces branches consistera à aller du point de repère vers l'une des deux branches dans le potentiel (sans exclure donc l'autre totalement, même dans les cas où le " potentiel » permet d'imaginer un état de choses vu comme pratiquement impossible : S'il était venu demain... se rapprochant ainsi de l'irréel), tandis que pour l'irréel, on partira par exemple $d u$ " haut» de la branche $p$ pour revenir au point de repère décroché et emprunter l'autre branche dont on sait qu'elle représente ce qui n'a pas été le cas. On rebrousse fictivement le chemin, avant d'emprunter l'autre, et de faire, à partir de ce repère, une inférence aboutissant à une autre prédication.

C'est ce même schéma dont la théorie des opérations énonciatives se sert pour décrire les différentes valeurs et usages des auxiliaires de modalité en anglais par exemple qui expriment également qu'on se situe hors de l'assertion (cf. Deschamps, 1999, Gresset, 1999) : les auxiliaires expriment des modes différents d'appréhension de l'altérité, à partir d'une position décrochée, suivant que l'autre branche est envisagée ou non et suivant la façon dont elle est envisagée.

\subsection{La composition de la protase et de l'apodose : relations interlexis}

Dans le domaine du fictif, au départ de la création du repère fictif, les deux branches sont toujours présentes, même si elles ne sont pas envisagées de la même façon suivant les cas (potentiel/ irréel). Cependant, la composition des deux prédications (la protase, représentée donc par une première bifurcation, et l'apodose inférée) complique nécessairement le problème puisque, à partir d'une des deux valeurs envisagées dans la protase, l'énonciateur fictif fait une projection imaginaire, qui, dans l'apodose, va être 
marquée comme plus ou moins probable (will / would, may/ might), et plus ou moins valuée dans le subjectif et l'intersubjectif.

Cette deuxième prédication va devoir être à son tour représentée par une bifurcation, et l'énonciateur va à nouveau avoir divers modes d'appréhension de l'altérité suivant qu'il construit une inférence forte, de l'ordre de la conséquence nécessaire (will / would), ou simplement plus ou moins possible (may/might par exemple). La composition des deux prédications par la relation d'inférence va intriquer le quantitatif de la nécessité, de la probabilité, de l'éventualité qui va moduler l'inférence, avec les valeurs qualitatives pouvant affecter, différemment, les deux domaines de l'irréel et du potentiel : regret, reproche, crainte, souhait, permission, suggestion plus ou moins appuyée, etc.

La relation interlexis entière se trouve alors affectée par ces valeurs qualitatives et prise dans une stratégie énonciative obéissant à une visée particulière. Si le quantitatif prime, on aura des argumentaires de type logique, et si le qualitatif prime (ce qui est très souvent le cas), on aura alors, en plus, toutes les valuations subjectives et intersubjectives citées précédemment, insérées dans des stratégies énonciatives très variées. On ne construit pas en général du fictif sans but énonciatif.

À partir de ce schéma très productif d'une première bifurcation, une " hypothétique », en ce qu'elle est une composition de lexis par inférence (de diverses natures), nécessite donc que l'on ajoute une deuxième bifurcation, orientée vers la deuxième prédication, q. En effet, cette deuxième prédication, construite à partir d'une deuxième notion complexe, fait elle aussi envisager nécessairement l'altérité quantitativement et qualitativement, puisqu'elle est issue d'une première altérité constitutive de la protase qui lui sert de repère, même si non q ou autre que q ne sont pas toujours envisagés. L'opération inverse marquée par exemple par sinon en français n'est jamais totalement oblitérée. On comprend qu'elle puisse si facilement se teinter de menace dans le domaine du potentiel.

Dans le dialogue suivant concernant de l'argent, extrait de La Traversée de Paris (Claude Autant-Lara, 1956), on voit comment Gabin refuse le fictif et la projection hypothétique construite sur le fictif, potentiel ou irréel, par Bourvil :

Bourvil : Tu me le proposerais, je refuserais.

Gabin : Comment tu peux savoir que tu refuserais puisque je ne te le propose pas?

En outre, il est bien sûr nécessaire de distinguer entre les types d'inférence et les rapports plus ou moins serrés entre $\mathrm{p}$ et $\mathrm{q}$, sémantiquement ou cognitivement. Il est par exemple des cas où $\mathrm{p}$ ne peut advenir que si q est validé (S'il n'y a pas de nuages, on pourra voir l'éclipse), des cas où $\mathrm{p}$ n'est pas une condition nécessaire à la réalisation de q (q conditionné par $\mathrm{p}$ : S'il n'y a pas de nuages, j'irai me promener). On voit qu'on rejoint les distinctions souvent faites entre condition et hypothèse ( $\mathrm{cf}$. de Vogüé ici même). La relation sémantique entre $p$ et $q$ est de nature différente dans chacun des exemples suivants :

S'il pleut, la route sera mouillée. Fais attention.

S'il pleut, j'irai au cinéma.

S'il pleut, ce sera encore de ma faute!

S'il pleut, tu trouveras un parapluie dans la voiture. ${ }^{5}$

\section{Diversité des formes}

La tentative d'unification de la représentation métalinguistique n'a pu être faite sans considérer des données empiriques, des énoncés en contexte. Dans un va-et-vient 
incessant entre données empiriques et théorisation, on ne peut ensuite que revenir aux énoncés attestables ou attestés. L'unification sémantique aide alors à sortir des énoncés hypotaxiques canoniques en si ou en if, pour considérer deux ordres de phénomènes :

- la multiplicité des formes que peuvent prendre la construction de repères fictifs dans une langue donnée et la construction de la relation interlexis ;

- la complexité de marqueurs tels que si, qui peut entrer dans ces schémas d'hypothétiques, mais qui se retrouve (cf. note 2) dans des concessives, des adversatives, des usages « austiniens », etc. Il s'agit d'en trouver la raison.

Par ailleurs, on le sait, le conditionnel présent ou passé en français, ou would en anglais, ne servent pas exclusivement à rendre cette valeur complexe. Mais les formes schématiques de ces marqueurs vont bien sûr permettre ces utilisations (cf. Merle, 1999 et ici même). Cette démarche onomasiologie et la recherche des formes schématiques des marqueurs ne sont pas notre objet ici' ${ }^{6}$.

La démarche qui semble donc aller de soi est de tenter de répertorier, ne serait-ce que dans une langue ou deux au départ, les marqueurs utilisés corrélativement, autrement dit fonctionnant en cooccurrence, dans la construction des repérages fictifs et des relations de composition avec les apodoses. Le terme "marqueurs » est utilisé ici pour renvoyer aux marqueurs segmentaux et suprasegmentaux, aux marqueurs d'ordre également protase anté- ou postposée -, ainsi qu'à « l'absence » de certains marqueurs dans les cas de parataxe.

\subsection{Les cas canoniques : hypotaxe}

On peut tout d'abord recenser et décrire formellement les cas les plus "canoniques " (simples) d'enchaînements protases / apodoses hypotaxiques (proposition « subordonnée » en si / if ou une locution conjonctive + proposition dite « principale » par exemple), quel que soit l'ordre (pertinent également) des deux propositions, dans leurs cooccurrences avec tels ou tels marqueurs aspecto-temporels dans les deux prédications, et avec tels ou tels types de procès (en français par exemple plus-que-parfait / imparfait / présent dans la protase, et les différentes formes du conditionnel ou du futur dans l'apodose, sans oublier que l'on n'a pas toujours les cas d'école plus-que-parfait / conditionnel passé, imparfait / conditionnel présent, présent / futur, mais que les «mélanges» dans les flexions verbales sont fréquents, dans le potentiel comme dans l'irréel, en liaison aussi avec les typologies de procès :

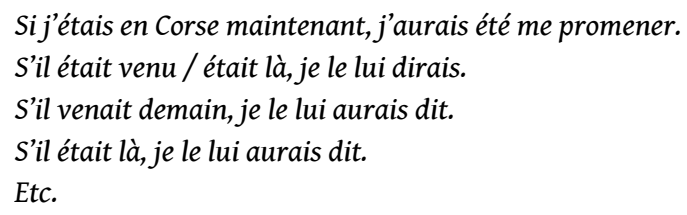

On oublie souvent des agencements comme :

S'il était venu demain, on aurait pu aller ensemble au cinéma. où les flexions verbales projettent ici des valeurs d'irréel sur l'à-venir, marquant $\mathrm{p}$ et $\mathrm{q}$ comme quasiment impossibles, et les valuant généralement en regret ou en soulagement. On voit, même avec des configurations hypotaxiques, les ambiguïtés souvent générées ici entre potentiel et irréel, quelles que soient les flexions verbales. On ne peut qu'en conclure que ce répertoire de marqueurs corrélatifs va devoir s'accompagner d'une recherche des indices variés du contexte (aussi différents que déterminants temporels 
déictiques, typologie des procès ou connaissance du monde par exemple) ou de la situation, qui vont déclencher telle ou telle interprétation (souvent modulée, valuée), ou laisser planer une ambiguïté, voulue ou non par l'énonciateur, perçue ou non par l'allocutaire.

\subsection{Protases protéiformes ${ }^{7}$ et asyndètes variées}

31 C'est au niveau des protases, ou de ce que l'on peut convenir d'appeler ainsi, quelles que soient leur catégorie et leur fonction dans un énoncé ou dans une relation inter-énoncés, que l'on trouve la plus grande variété, et que se pose le problème des formes que peut prendre une notion complexe érigée en protase.

On trouve en effet, notamment en français, une grande variété de marqueurs associés à la composition des deux prédications. Les protases peuvent être plus ou moins explicitement marquées comme telles, ou "tronquées", marquées par telle ou telle flexion verbale, dans une imbrication hypotaxique ou parataxique. Elles peuvent revêtir des formes impératives ou interrogatives. Elle peuvent aussi avoir des apodoses implicites, être elles-mêmes implicites, à reconstruire dans le contexte amont.

Soient les deux familles paraphrastiques (à protase antéposée uniquement et à marqueur temporel déictique univoque) :

Potentiel :

Si elle vient demain, elle résoudra tous les problèmes.

Si elle venait demain, elle résoudrait tous les problèmes.

Si elle était venue demain, elle aurait résolu tous les problèmes.

Qu'elle vienne demain, et elle résoudra tous les problèmes / et tous les problèmes seront

résolus 8 .

Viendrait-elle demain qu'elle résoudrait tous les problèmes.

A supposer qu'elle vienne demain, elle résoudra / résoudrait tous les problèmes.

Pour peu qu'elle vienne demain, elle résoudrait tous les problèmes.

Imaginons qu'elle vienne demain : elle résoudrait tous les problèmes.

Et si elle venait demain? Ça résoudrait tous les problèmes.

Elle viendrait demain, elle résoudrait tous les problèmes.

Elle vient demain, elle résout tous les problèmes.

Elle vient demain et tous les problèmes sont / seraient résolus.

Avec elle ici demain, tous les problèmes seraient résolus 9 .

Son arrivée demain résoudrait tous les problèmes.

Présente demain, elle résoudrait tous les problèmes.

L'avoir ici demain résoudrait tous les problèmes.

Elle ici demain, plus de problèmes.

Etc.

Irréel :

Sij'avais eu le roi de trèfle hier, je l'aurais faite (ma partie).

Sij'avais eu le roi de trèfle hier, je la faisais.

Sij'avais le roi de trèfle hier, je la faisais.

J'eusse eu le roi de trèfle hier, je l'eusse faite.

Pour peu que j'aie eu le roi de trèfle hier, je la faisais / je l'aurais faite.

Aurais-je eu le roi de trèfle hier, que je l'aurais faite.

Eussé-je eu le roi de trèfle hier, je l'eusse faite.

J'aurais eu le roi de trèfle hier; je la faisais.

J'aurais eu le roi de trèfle hier, je l'aurais faite.

J'avais le roi de trèfle hier, je la faisais.

Avec le roi de trèfle hier, je la faisais.

Avec le roi de trèfle hier, je l'aurais faite. 
Moyennant le roi de trèfle hier, je la faisais / je l'aurais faite.

Il suffisait que j'aie le roi de trèfle hier et je la faisais /je l'aurais faite.

Il suffit que j'aie le roi trèfle hier et je la fais.

Avoir le roi de trèfle hier, c'était la faire / ç'aurait été la faire.

Le roi de trèfle hier me la faisait faire / me l'aurait fait faire.

Le roi de trèfle (présent) dans mon jeu hier, je la faisais / je l'aurais faite.

Le roi de trèfle hier et je la faisais.

? Roi de trèfle, partie gagnée ${ }^{10}$.

Etc.

les deux domaines de l'irréel et du potentiel sémantiques notamment), permet en effet, parce qu'elle la dépasse par l'abstraction de la représentation métalinguistique, d'aborder la diversité des marques et de leurs agencements. Les analyses distributionnelles, indispensables dans un premier temps, sont ici régies par des interprétations sémantiques : y a-t-il ou non création (dans la construction du sens) et interprétation (dans sa reconstruction), par décrochage, d'un repérage fictif, quel qu'il soit? Quand bascule-t-on dans le fictif et avec quels indices? Et dans quelle sorte de fictif?

L'étude spécifique des formes schématiques des différents marqueurs dans une langue donnée (conditionnel, imparfait, conjonctions et locutions conjonctives, nominalisations, diathèse, typologie des procès, personnes, etc.), et leurs corrélations en cooccurrence, devrait permettre de rendre compte des effets de sens qui prolifèrent à partir de ce schéma de base. Ce n'est pas notre propos ici. 


\subsection{Réflexion sur la syntaxe et la sémantique de la " subordination »}

41 Les "hypothétiques» articulent deux éléments, deux prédications, ou pseudoprédications (groupes prépositionnels, groupes nominaux /nominalisés par exemple en protases), et remettent en cause les catégories grammaticales traditionnelles et leurs fonctions possibles (en particulier les « circonstancielles »).

En effet, les protases réalisées en groupes participiaux, ou nominaux ont une incidence sur l'unique relation prédicative présente alors explicitement :

Avec le roi de trèfle, je la faisais.

Sa venue résoudrait tous les problèmes.

et l'apodose ne peut plus avoir d'individualité syntaxique apparente nette.

Ceci n'est pas spécifique aux hypothétiques. Ce serait aussi le cas dans l'effectif (par exemple Avec le roi de trèfle je l'ai faite). Le prédicat d'existence localisé avec le roi de trèfle renvoie bien à une lexis qui donne accès à une autre lexis. Les gloses en subordonnée + principale(Si j'avais eu le roi de trèfle, pour le fictif, Comme / quand j'ai eu le roi de trèfle, pour l'effectif) affleurent vite, mais ne résolvent pas pour autant tous les problèmes. La composition de la consécution et de la concomitance est toujours présente. Les notions complexes évoquées sont mises dans le même domaine, en contiguïté et en continuité donc.

Dans le domaine du fictif, on pense aussi à des gloses / paraphrases comme :

Je n'avais pas le roi de trèfle. Sinon je l'aurais faite.

qui posent le problème des liens entre hypotaxe et parataxe d'une manière différente par rapport à l'énoncé :

J'avais le roi de trèfle hier, je la faisais.

où le premier " élément » (J'avais le roi de trèfle hier), ne peut être énoncé seul sans faire basculer le sens vers l'effectif, et donc l'inverser. Les énoncés réellement parataxiques, comme le dernier cité par exemple où il y a une symétrie formelle entre les deux "éléments ", ne peuvent que rarement servir de glose à des énoncés hypotaxiques qui, eux, permettent souvent de s'affranchir de l'iconicité de la consécution extralinguistique, et qui ouvrent moins de champ aux ambiguiités ${ }^{11}$.

Les "hypothétiques" posent, elles aussi, le difficile problème d'une théorie de la subordination, appréhendée traditionnellement au niveau syntaxique (qu'on parle de «principale» et de «subordonnée », ou d'« imbricante» et d'«imbriquée ») : elles montrent bien que la théorie doit rendre compte de l'enchevêtrement des opérations énonciatives de repérage avec les opérations prédicatives.

En effet, la prédication antéposée, en si par exemple, sert, on l'a vu, de repère à la prédication exprimant l'apodose, on l'a vu. Ce qui est, au niveau syntaxique, la "principale» est, au niveau énonciatif, subordonné au repérage établi par la " subordonnée ». On retrouve certains des problèmes liés à la "subordination inverse ». Nous ne creuserons pas plus avant ce champ complexe (cf. Wyld, 1998, pour une théorisation partielle de la subordination dans le cadre d'une théorie des opérations énonciatives).

Se posent donc toute une série de questions, ne serait-ce que dans les domaines du potentiel et de l'irréel, questions que je me contenterai de passer en revue ici :

- celle des liens entre antéposition et postposition des protases ; 
- celle des liens à élucider entre hypotaxe et (différentes formes de) parataxe - problème particulièrement pertinent en français, et aux types d'ambiguïtés qui s'y attachent ;

- celle des marqueurs suprasegmentaux (les énoncés parataxiques n'ayant pas toujours des contours intonatifs spécifiques ${ }^{12}$ ) marquant l'inférence et soulignant les valuations positives ou négatives ;

- celle de la nature des relations (syntaxiques ?) établies entre protase et apodose quand les protases sont réalisées par des groupes prépositionnels, ou nominaux, ou encore adjectivaux ;

- celle des protases implicites, que l'on peut reconstruire par le contexte amont, ou la situation, et notamment celle des autres formes d'irréel ( $\mathrm{Tu}$ aurais pu y aller quand même, You could have done it, You might have been killed, etc.) ou de potentiel (Si seulement il venait! Il se pourrait qu'il vienne, etc.), dont on peut rendre compte par une utilisation similaire du schéma de bifurcation (Deschamps, 1999) et par une prise en compte des formes schématiques des marqueurs impliqués en corrélation.

Les réponses à ces questions complexes aideraient à cerner les raisons de l'ambiguïté de certains énoncés, notamment parataxiques, des relations de paraphrases, et aussi de l'irrecevabilité de certains énoncés. On en connaît déjà certaines.

\section{Cas d'ambiguïté}

49 L'interprétation sémantique de marqueurs polysémiques et de configurations, qui laissent parfois ouvertes des potentialités d'ambiguïté, les phénomènes de rétroaction dans la reconstruction du sens, les divers va-et-vient de relations entre marqueurs et préconstruits, posent le problème de l'étude des indices contextuels d'interprétation, des filtres construits par le frayage contextuel et des inductions qu'ils autorisent. Le contexte, filtre interprétatif, est donc générateur d'interprétation (Kleiber, 1997) et évite souvent les ambiguïtés, tout comme les connaissances plus ou moins partagéesde différents ordres sur lesquelles s'appuie la "noria sémantique» dont parle Culioli. Les formes schématiques dégagées pour certains marqueurs seront suffisamment générales pour se prêter aux déformations ordonnées par les marqueurs co-présents, que ce soit des marqueurs lexicaux, ou grammaticaux, notamment aspecto-temporels, des courbes intonatives, des ancrages situationnels, déictiques par exemple, etc.

50 Cependant, quel que soit le frayage construit par le contexte, on doit tenter de rendre compte des ambiguïtéspermises par certaines associations et non, ou moins, par d'autres.

51 J'ai déjà mentionné l'ambiguïté fréquente, hors marqueurs déictiques, de nombre des exemples des familles de paraphrases citées plus haut. L'ambiguïté se jouait soit entre valeurs de potentiel et irréel croisées avec référence au révolu ou à l'actuel vs l'à-venir par exemple: Si elle était venue, elle aurait résolu tous les problèmes, qui peut être énoncé en cooccurrence avec les déictiques hier ou demain, soit entre fictif (irréel) et non fictif (itératif) : J'avais le roi de trèfle, je la faisais. Le contexte ou la situation vont la plupart du temps filtrer une des deux interprétations, mais il faut aussi comprendre pourquoi un tel énoncé permet les deux interprétations en fictif et en effectif itératif.

Soit un énoncé similaire cité et commenté par Culioli :

Il me téléphonait, je venais.

(où la transcription comportait une virgule). 
Cet énoncé, hors contexte du moins, et à l'écrit, présente une ambiguïté dont il faut rendre compte, entre itération et fictif (irréel du passé) :

Chaque fois qu'il me téléphonait, je venais.

S'il m'avait téléphoné, je serais venu.

Si, le plus-que-parfait et le conditionnel passé enlèvent ici toute ambiguïté entre l'itératif et l'irréel.

Il faut rendre compte de ces emplois de l'imparfait dans des énoncés parataxiques qui présentent cette ambiguïté entre itération et irréel. Les imparfaits instaurent ici deux différents modes de mise à distance :

- une mise à distance par une position hors du temps, ici hors de la classe ordonnée des instants pour l'itératif, où le complémentaire n'est pas pris en compte; on ne peut pas considérer chaque événement dans sa spécificité. On envisage tous les événements. On a une valeur de générique ;

- et une mise à distance par décrochage et distinction binaire effectif / fictif pour l'irréel par rapport à un repère fictif, avec donc prise en compte du complémentaire, un possible qui n'a pas eu lieu et qui évoque le complémentaire validé dans le révolu (il ne m’a pas téléphoné). Ici non plus on ne travaille pas dans le repérage temporel, mais sur la superposition de deux états de choses : ce qui a eu lieu par opposition à ce qui n'a pas eu lieu, mais est envisagé.

Mais avec l'interprétation itérative comme avec l'interprétation fictive, on touche aux relations de consécution (il suffisait qu'il me téléphone pour que je vienne, nécessairement, automatiquement il s'ensuivait que...), et on dévoile d'autres modalités enfouies.

Le paramètre du temps est donc quand même activé en vue d'établir une relation de l'ordre du raisonnement, c'est-à-dire en fait de l'ordre du paramètre subjectif des coordonnées énonciatives, prépondérant dans le fictif. L'énoncé :

Tu me téléphonais, je venais.

sera moins ambigu, à cause de la deuxième personne. On l'interprétera préférentiellement comme renvoyant à un irréel.

56 L'énoncé S'il me téléphonait, je venais ne peut que prendre l'interprétation de renvoi à l'effectif itératif, générique. Le rajout de si avec deux imparfaits change le sens pour faire basculer dans le générique (quand, chaque fois que).

L'ambiguïté peut aussi fonctionner à un autre niveau (cf. Merle, ici même). Soit en effet l'énoncé :

S'il avait fait beau, elle serait allée se promener.

(troisième personne et non première, pour laisser ouverte l'ambiguïté)

Cet énoncé est ambigu, même si son apparence de cas d'école le fait d'abord sans doute interpréter, hors indices contextuels autres, comme relevant de l'irréel du passé: l'énonciateur sait qu'il n'a pas fait beau, ou qu'il ne fait pas beau (assertion préconstruite sur le révolu ou l'actuel); c'est le cas que non $p$, j'imagine si p et l'inférence, qui entraîne $q$, fondée sur les connaissances que l'on a du comportement habituel de la femme.

Mais il y a une deuxième interprétation possible selon laquelle l'énonciateur fait une reconstruction logique à partir d'un autre préconstruit : c'est le cas qu'elle n'est pas allée se promener, et c'est donc qu'il n'a pas fait beau. Le fait qu'il n'a pas fait beau, ou qu'il ne fait pas beau n'est pas un préconstruit existentiel ; sa reconstruction du fait qu'il n'a pas fait beau est fondée sur la connaissance qu'a l'énonciateur des comportements de la femme. Il sait que non q est le cas et il en infère q donc $p$ : s'il avait fait beau / si c'était 
vrai qu'il avait fait beau, elle serait allée se promener. Or elle n'est pas allée se promener. Donc c'est qu'il n'a pas fait beau.

On n'a pas le même type d'opérations sous-jacentes. On ne part pas des mêmes préconstruits existentiels, frayés sans doute par le contexte, et on n'est pas dans la même intersubjectivité argumentative : dans le deuxième cas on fait une démonstration, et la représentation que l'on a du coénonciateur n'est pas la même.

61 Voici un autre exemple intéressant d'ambiguïté potentielle entre l'effectif et le fictif à partir des protases in war et with no one to need her (cf. Trévise, à paraître) :

The angel, that is how she is sometimes known. The Angel in Black who comes to save the children of the brown folk from their croups and fevers. (...) Night after night she sits up with whimpering children or women in labour, fighting off sleep. (...) Her heart sings. In war she would lighten the last hours of the wounded. They would die with smiles on their lips, gazing into her eyes, clasping her hand. Her stores of compassion are boundless. She needs to be needed. With no one to need her she is baffled and bewildered. Does that not explain everything? (J. M. Coetzee, In the Heart of the Country, 1977, p. 5)

\section{Et si on concluait ? (*Si on concluait ?)} dynamique des opérations sous-jacentes liées à la construction de l'irréel et du potentiel, dans ce qu'ils ont de commun, et de spécifique (l'un par rapport à l'autre mais aussi par rapport aux autres formes de relations interprédicatives d'inférence comme la concession - cf. Filippi-Deswelle, 1998, et ici même, et Ranger, 1999 -, les argumentations rhétoriques diverses, etc.). Ces représentations métalinguistiques sont à associer à l'apparition ou non de marqueurs de différents types, agencés différemment du point de vue syntaxique. La théorie de opérations énonciatives tente de rendre compte de la déformabilité des configurations signifiantes, par laquelle se manifestent les déploiements du sens.

sagit alors de relier cette représentation de la mouvance et de la dynamique d'ajustements à l'épaisseur des mots, aux formes schématiques (si / if par exemple) ainsi qu'aux indices contextuels (amont et aval) permettant d'induire telle ou telle interprétation, ou laissant ouverte une ambiguïté, même si on intègre les frayages contextuels et / ou les relations physico-culturelles dites primitives. Les ajustements concernent aussi, on l'a vu, les valuations personnelles des sujets énonciateurs dans leurs échanges, et donc tout le domaine de l'intersubjectif (construction de valuations positives ou négatives, représentations des préconstruits du coénonciateur et des ajustements aux préconstruits supposés de l'autre, etc.).

On ne peut échapper à des considérations sur les relations entre hypotaxe et parataxe, ni à l'analyse des différents types de liens en termes de dépendance ou d'autonomie entre les « éléments » parataxiques. vertigineuse.

Le travail effectué sur les unités par l'activité de langage est un travail de construction / reconstruction de sens au fur et à mesure de la linéarité de la production/ reconnaissance d'énoncés par des actes d'énonciation opérés par des individus. Les différents niveaux interagissent, et on n'est plus dans la linéarité mais dans l'épaisseur et l'intrication des relations. Le sens ne naît pas de l'addition des unités, mais dans la prise en compte de va-et-vient linéaires amont / aval, aval / amont, avec l'épaisseur des mots 
et des constellations qu'ils comportent, avec aussi l'épaisseur des inférences et plus généralement de toutes les représentations partagées ou plus subjectives.

Deux citations pour clore, très provisoirement, cette réflexion :

Cette dynamique de construction du sens dans l'énoncé implique de ne pas prendre la catégorisation linguistique comme une catégorie mentale fixe mais seulement comme un élément intervenant à un certain niveau de construction des représentations linguistiques. Entre la pensée et le langage, comme entre les unités et l'énoncé, il y a toute une dynamique de construction qui passe par des ajustements, des approximations et d'éventuels ratages.

De plus, cette construction progressive du sens dans l'énoncé est caractérisée par une non linéarité des effets de sens. Celle-ci provient d'une rétroaction constante des unités les unes sur les autres, ce qui suppose des mécanismes cognitifs de type « réentrant » qui restent encore à étudier (Robert, 1997 : 37).

(...) non pas un système en tout ou rien, mais un système complexe, ou interagissent des sous-systèmes hétérogènes ( $d$ 'où par exemple, la superposition des représentations pour un même marqueur), où l'on opère sur des domaines, des champs, des repérages, des orientations, où les représentations se construisent et se transforment. Sans un sujet, dans ses relations avec d'autres sujets, on ne peut rendre compte des observations empiriques qui sont la trace de notre activité cognitive, telle qu'elle apparaît, de façon spécifique, à travers le langage.

(...) Seul un système qui choisit d'aborder les étagements de formes, les déformations, la prolifération, bref un système topologique et dynamique, et seul le concept d'invariance permettent d'analyser de façon cohérente la singularité des langues en relation avec l'universalité du langage (Culioli, 1997 : 56).

On voit les ajustements nécessaires pour interpréter l'échange suivant par exemple:

- A : Dis-moi, au fait, ton père, s'il était toujours vivant, ça lui ferait quel âge maintenant?

- B : Oh mon père, s'il était toujours vivant, il serait mort depuis longtemps !13

\section{BIBLIOGRAPHIE}

CHARREYRE, C. (1984), « Quand MIGHT (HAVE -EN) peut se traduire par c'était comme si », in Cahiers Charles V, 6, Paris, Ophrys, pp. 27-58.

CULIOLI, A. (1990), Pour une linguistique de l'énonciation, tome 1, Paris, Ophrys.

CULIOLI, A. (1997), « Subjectivité, invariance et déploiement des formes dans la construction des représentations linguistiques ",in Diversité des langues et représentations cognitives, Paris, Ophrys, pp. 43-57.

CULIOLI, A. (1999), Pour une linguistique de l'énonciation, tome 3, Paris, Ophrys.

DESCHAMPS, A. (1999), « Essai de formalisation du système modal de l'anglais », in Les opérations de détermination : quantification / qualification, Paris, Ophrys, pp. 269-285.

FEUILLET, J. (1993), « L'hypothèse », in Subordinations / Subordination, CERLICO, 6, pp. 67-96.

FILIPPI, C. (1997), « THOUGH et la relation de concession : opération énonciative et jeu sur le domaine notionnel », in La notion, Paris, Ophrys, pp. 91-98. 
FILIPPI-DESWELLE, C. (1998), La relation dite de « concession » : étude de THOUGH, ALTHOUGH, EVEN THOUGH et EVEN IF antéposés en anglais contemporain, Thèse de Doctorat, Université Paris 7.

GRESSET, S. (1999), CAN / MAY, MAY / MIGHT et MIGHT / COULD, ou l'interchangeabilité en question, Thèse de Doctorat, Université Paris $\mathrm{X}$ - Nanterre.

KLEIBER, G. (1997), « Quand le contexte va, tout va et... inversement », in Cl. GUIMIER (ed.), Cotexte et calcul du sens, Presses Universitaires de Caen, pp. 11-29.

MERLE, J.-M. (1998), « De la projection hypothétique à l'atténuation : conditionnel français vs WOULD + BV », in Linguistique contrastive et traduction, 4, Paris, Ophrys, pp. 115-185.

MERLE J.-M. (1999), Etude du conditionnel et de ses traductions en anglais, Thèse de Doctorat, Université Paris 7.

RANGER, G. (1998), Les constructions concessives en anglais : une approche énonciative, Cahiers de Recherche, numéro spécial, Paris, Ophrys.

ROBERT, S. (1997), « Variation des représentations linguistiques : des unités à l'énoncé », in Diversité des langues et représentations cognitives, Paris, Ophrys, pp. 25-39.

TREVISE, A. (à paraître), « Effectif et fictif : quelques cas d'ambiguïté ».

de VOGÜÉ, S. (1987), « La conjonction si et la question de l'homonymie », BULAG 13, Université de Besançon, pp. 105-189

de VOGÜÉ, S. (1992), «Si, la syntaxe et le point de vue des opérations », in La théorie d'Antoine Culioli. Ouvertures et incidences, Paris, Ophrys, pp. 123-144.

WYLD, H. (1998), Problèmes de subordination relatifs à l'expression des valeurs aspectuo-temporelles, modales et causales en anglais contemporain : une approche énonciative, Thèse de Doctorat, Université Paris 7.

\section{NOTES}

1. On se reportera pour une appréhension plus large du domaine notamment aux écrits, cités dans les références bibliographiques, de Culioli (notamment le tome 3 de Pour une linguistique de l'énonciation, 1999), de Vogüé, Merle, Feuillet, mais aussi aux travaux sur la concession en anglais (Filippi-Deswelle, Ranger). Il serait trop long d'en rendre compte ici, mais certains pans du domaine ont déjà été amplement travaillés, tant au niveau des fonctionnements de marqueurs polyvalents, qu'au niveau d'une représentation abstraite (métalinguistique) des opérations en jeu.

2. Il semble souvent utile de rappeler que le coénonciateur n'est pas l'allocutaire, mais la représentation que s'en construit l'énonciateur.

3. cf. Wyld, 1998.

4. cf. Deschamps, 1999.

5. Nous n'avons ici exploré que les cas clairs de potentiel ou d'irréel. On se reportera par exemple à l'article de $\mathrm{S}$. de Vogüé ici même et aux différentes références bibliographiques pour mesurer la complexité des schémas sémantiques qui peuvent être convoqués.

6. Mais le domaine bénéficie désormais de travaux importants sur un certain nombre de marqueurs, notamment en anglais et en français ainsi que dans le domaine spécifique de la contrastivité (cf. les écrits de Culioli, Merle, de Vogüé, Filippi ou Ranger, cités dans la bibliographie).

7. Pour reprendre l'expression de Merle, 1998. 
8. On se reportera à Culioli (1999: 160-163) pour une analyse de et et de ou dans ce type de contexte, et en particulier pour une analyse des énoncés :

Qu'il téléphone et je viens.

Qu'il téléphone ou je ne viens pas.

9. Dans ces deux derniers exemples, on voit les liens (complexes) entre diathèse dans l'apodose, type de repérage construit dans la protase, et thématisation / frayage opérés par le contexte amont. L'énoncé à l'actif :

? Elle vient demain et elle résoudrait tous les problèmes. me semble problématique.

10. cf. Un coup de barre, Mars et ça repart.

11. cf. la non conformité sémantique des énoncés suivants par rapport aux énoncés cités précédemment :

* Je la faisais, j'avais le roi de trèfle.

* Je l'aurais faite, j'aurais eu le roi de trèfle.

* Je la faisais, j'aurais eu le roi de trèfle.

* Je l'aurais faite, j'avais le roi de trèfle.

? J'avais le roi de trèfle et je la faisais.

12. Il semble que la courbe mélodique (et l'absence de pause) soient identiques dans les exemples:

Si j'avais eu le roi de trèfle, je l'aurais faite.

J'aurais eu le roi de trèfle, je l'aurais faite.

J'avais le roi de trèfle, je la faisais.

Avec le roi de trèfle, je la faisais / je l'aurais faite.

(d'ou la gêne qu'on éprouve à mettre un virgule à l'écrit pour les deux derniers exemples, si elle est censée représenter une pause, et ne pas uniquement permettre une meilleure lisibilité ; en français on met volontiers une virgule dans les cas «canoniques » de subordonnée + principale, ce qui n'est pas toujours le cas, faut-il le rappeler, en anglais).

13. Merci à Rémy Porquier pour cet exemple authentique.

\section{AUTEUR}

\section{ANNE TRÉVISE}

Université de Paris X-Nanterre

U.F.R. d'Etudes anglo-américaines

200, avenue de la République

92000 - Nanterre Cedex

trevise.anne@paris7.jussieu.fr

Groupe de recherche Jan Comenius 